\title{
Robust optical readout and characterization of nuclear spin transitions in nitrogen-vacancy ensembles in diamond
}

\author{
A. Jarmola $\odot,{ }^{1,2,{ }^{*}}$ I. Fescenko ${ }^{\circ},{ }^{3}$ V. M. Acosta, ${ }^{3}$ M. W. Doherty $\odot,{ }^{4}$ F. K. Fatemi, ${ }^{2}$ T. Ivanov ${ }^{2}$ \\ D. Budker ${ }^{1},{ }^{1,5}$ and V. S. Malinovsky ${ }^{2}{ }^{2}$ \\ ${ }^{1}$ Department of Physics, University of California, Berkeley, California 94720, USA \\ ${ }^{2}$ U.S. Army Research Laboratory, Adelphi, Maryland 20783, USA \\ ${ }^{3}$ Center for High Technology Materials and Department of Physics and Astronomy, University of New Mexico, \\ Albuquerque, New Mexico 87106, USA \\ ${ }^{4}$ Laser Physics Centre, Research School of Physics, Australian National University, Canberra 2601, Australia \\ ${ }^{5}$ Helmholtz-Institut Mainz, GSI Helmholtzzentrum für Schwerionenforschung, 55128 Mainz, Germany
}

(Received 31 December 2019; revised manuscript received 25 February 2020; accepted 23 March 2020; published 28 April 2020)

\begin{abstract}
Nuclear spin ensembles in diamond are promising candidates for quantum sensing applications, including rotation sensing. Here we characterize the optically detected nuclear spin transitions associated with the ${ }^{14} \mathrm{~N}$ nuclear spin within diamond nitrogen-vacancy (NV) centers. We observe that the contrast of the nuclearspin-dependent fluorescence is comparable to the contrast of the NV electron-spin-dependent fluorescence. Using Ramsey spectroscopy, we investigate the temperature and magnetic field dependence of the nuclear spin transitions in the 77.5-420 K and 350-675 G range, respectively. The nuclear quadrupole coupling constant $Q$ was found to vary with temperature $T$, yielding $d|Q| / d T=-35.0(2) \mathrm{Hz} / \mathrm{K}$ at $T=297 \mathrm{~K}$. The temperature and magnetic field dependencies reported here are important for quantum sensing applications such as rotation sensing and potentially for applications in quantum information processing.
\end{abstract}

DOI: 10.1103/PhysRevResearch.2.023094

\section{INTRODUCTION}

Quantum sensors based on nitrogen-vacancy (NV) spin qubits in diamond are used in a number of sensing modalities, including magnetometry, electrometry, and thermometry [1-4]. Typically, the qubit used for sensing applications is formed from the NV electron spin levels due to their high sensitivity to environmental perturbations. However, nuclear spins can be more suitable for applications where sensitivity to magnetic noise and temperature variations is undesirable, such as rotation sensing [5-7]. Of particular interest are nitrogen nuclear spins intrinsic to NV centers. These spins can be efficiently optically polarized and read out via NV electron spins.

Consider the example of using the intrinsic ${ }^{14} \mathrm{~N}$ nuclear spins of an ensemble of NV centers for rotation sensing. The ${ }^{14} \mathrm{~N}$ nuclear spins are prepared in a superposition state and precess about their quantization axis with nuclear precession rate $\omega_{0}$. If the diamond rotates about this axis with a rate $\omega$, the nuclear precession rate in the diamond reference frame is

\footnotetext{
*jarmola@berkeley.edu

Published by the American Physical Society under the terms of the Creative Commons Attribution 4.0 International license. Further distribution of this work must maintain attribution to the author(s) and the published article's title, journal citation, and DOI.
}

$\omega_{0}-\omega$. The minimum detectable change in $\omega$ is given by

$$
\delta \omega \approx \frac{1}{C \sqrt{\eta N T_{2}^{*} \tau}},
$$

where $C$ is the fractional contrast of spin-state-dependent fluorescence, $\eta$ is the photon-collection efficiency, $N$ is the number of interrogated spins, $T_{2}^{*}$ is the spin-coherence time, and $\tau$ is the total integration time. From Eq. (1), it can be seen that intrinsic ${ }^{14} \mathrm{~N}$ nuclear spins offer an advantage over electron spins owing to their $10^{3}$-fold longer coherence time [8] at the same number density. Nuclear spins also benefit from a $10^{3}-10^{4}$ times smaller gyromagnetic ratio than that of electron spins, minimizing the frequency shifts due to fluctuations in the magnetic field.

A remaining challenge is to realize a high spin readout contrast $C$ without introducing additional sources of technical noise. One avenue that has been explored is the use of conditional microwave pulses to map nuclear spin states onto NV electron spin states, which requires the use of both radio-frequency and microwave fields [9-11]. This approach has been shown [8] to achieve a readout contrast of $C \gtrsim$ $10^{-2}$, approaching the contrast realized with NV electron spin ensembles [4]. However, environmental influences, such as magnetic field and temperature variations, affect the electron spin transition frequency, limiting the robustness of this technique [8].

Optical readout of the nuclear spin state can also be accomplished directly without the use of microwave 
mapping pulses near the excited-state level anticrossing (ESLAC) [8-10]. The advantage of this technique is that it directly provides information about the nuclear spin states without precise knowledge of the electron spin transition frequencies. While this technique has been previously demonstrated, here we systematically analyze its readout contrast.

In this work, we characterize the optical readout mechanism of ${ }^{14} \mathrm{~N}$ NV nuclear spin ensembles. We find that the contrast of nuclear spin Rabi oscillations exceeds $2 \%$ in a broad range of magnetic fields, from approximately 450 to $550 \mathrm{G}$. Using Ramsey spectroscopy, we investigate the temperature and magnetic field dependence of the nuclear spin transition frequencies. At $297 \mathrm{~K}$, we find that the temperature dependence of the nuclear quadrupole coupling constant is $d|Q| / d T=-35.0(2) \mathrm{Hz} / \mathrm{K}$, which is about 2000 times smaller than the temperature dependence of the NV electron spin zero-field splitting $D$ [12]. Our results hold promise for quantum sensing applications requiring minimal magnetic field and temperature dependence, including gyroscopes and clocks [13].

\section{OPTICAL ADDRESSING OF THE ${ }^{14} \mathrm{~N}$ NUCLEI}

A schematic of the relevant energy levels and transitions in diamond NV centers is presented in Fig. 1(a); see, also, Appendix A. Application of light with wavelength shorter than that of the zero-phonon line of the ${ }^{3} A_{2} \rightarrow{ }^{3} E$ transition (at $637 \mathrm{~nm}$ ) optically induces spin polarization of the NV centers into the $m_{S}=0$ sublevel of the ground electronic state [14]. If a magnetic field $\mathbf{B}$ is applied along the axis of the $\mathrm{NV}$ center, the $m_{S}= \pm 1$ sublevels of the ground and excited states experience Zeeman shifts. At $B \approx 500 \mathrm{G}$, the $m_{S}=0$ and $m_{S}=-1$ sublevels in the excited state become nearly degenerate. This condition is referred to as the excited-state level anticrossing (ESLAC). Near the ESLAC, electron spin polarization is effectively transferred to the nuclei, achieving a nearly complete ${ }^{14} \mathrm{~N}$ polarization under a wide range of magnetic fields. Nuclear spin polarization at the ESLAC mediated by NV centers in diamond has been described in Refs. $[9,10,15,16]$ and is only briefly summarized here. Near the ESLAC, strong hyperfine coupling in the excited state allows the energy-conserving electron-nuclear spin flip-flop processes to occur between coupled electron-nuclear spin states, denoted $\left|m_{S}, m_{I}\right\rangle$. Specifically, such processes can lead to flip flops between $|0,-1\rangle$ and $|-1,0\rangle$, as well as between the $|0,0\rangle$ and $|-1,+1\rangle$ states; see Figs. 1(b) and 1(c). Under optical illumination near the ESLAC, the system is polarized into the $|0,+1\rangle$ spin state; see Fig. 1(d).

The mechanism responsible for nuclear spin polarization leads to nuclear-spin-dependent fluorescence and provides the means for direct nuclear spin optical readout. The polarized $|0,+1\rangle$ spin state produces maximum fluorescence because it is not affected by mixing in the excited state and does not pass thought the dark singlet states as often. As depicted in Figs. 1(b) and 1(c), respectively, the $|0,-1\rangle$ and $|0,0\rangle$ states undergo an electron-nuclear spin flip-flop process in the excited state, which changes their electron spin projection to $m_{S}=-1$ and causes them to pass through the dark singlet states, which reduces their fluorescence. The degree of mixing in the excited state, and therefore the fluorescence rate, is

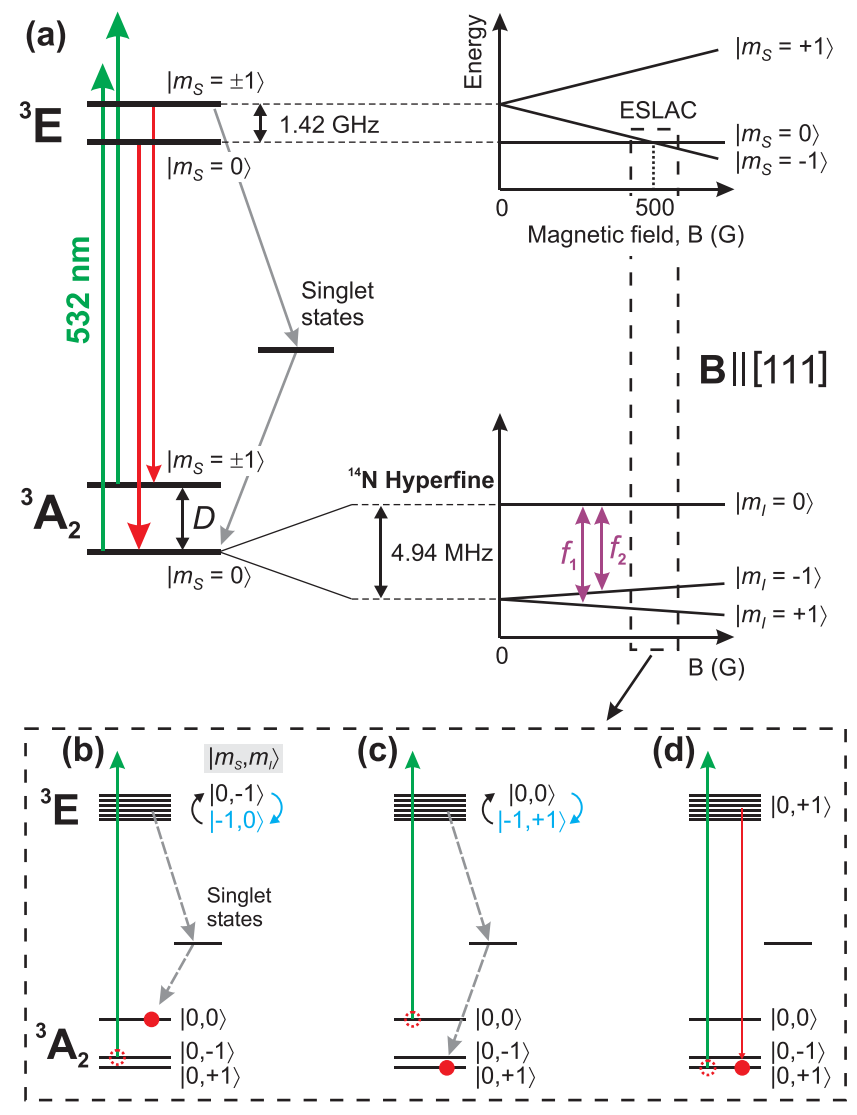

FIG. 1. (a) Energy levels of the NV center and their dependence on the magnetic field $\mathbf{B}$ applied along the NV symmetry axis. The interaction of the intrinsic electric-field gradient with the quadrupole moment of the ${ }^{14} \mathrm{~N}$ nucleus (nuclear spin $I=1$ ) leads to a $\sim 4.94 \mathrm{MHz}$ hyperfine splitting in the $m_{S}=0$ groundstate manifold. In the presence of an applied magnetic field, the $m_{I}= \pm 1$ nuclear levels split. At $\sim 500 \mathrm{G}$, the $m_{S}=0$ and $m_{S}=-1$ levels within the excited state undergo a level anticrossing (ESLAC). (b)-(d) Optical pumping at the ESLAC leads to polarization of the ${ }^{14} \mathrm{~N}$ nuclear spin intrinsic to the $\mathrm{NV}$ center into the $m_{I}=+1$ state. The mechanism responsible for nuclear spin polarization leads to nuclear-spin-dependent fluorescence.

different for the $|0,0\rangle$ and $|0,-1\rangle$ states and depends on the applied magnetic field (Appendix B).

\section{EXPERIMENTAL METHODS}

We used a custom-built confocal-microscopy setup to measure optically detected nuclear magnetic resonances (ODNMRs) in an ensemble of NV centers. The sample used in our experiments is a [100]-cut high-pressure high-temperature grown diamond with an initial nitrogen concentration of $\sim 50 \mathrm{ppm}$. NV centers were created by irradiating the sample with $10 \mathrm{MeV}$ electrons at a dose of $\sim 10^{18} \mathrm{~cm}^{-2}$ and subsequent annealing in vacuum at $800^{\circ} \mathrm{C}$ for three hours.

The diamond sample was mounted inside a continuousflow microscopy cryostat. Pulses of $532 \mathrm{~nm}$ laser light ( $20 \mathrm{~mW}, 20 \mu \mathrm{s}$ duration) were focused onto the diamond using a microscope objective with 0.6 numerical aperture. Fluorescence was collected through the same objective, passed 


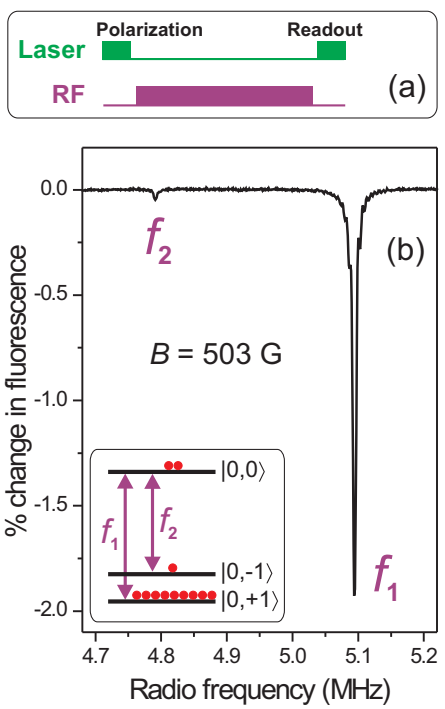

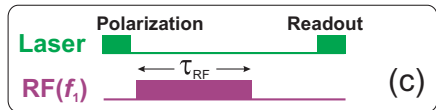
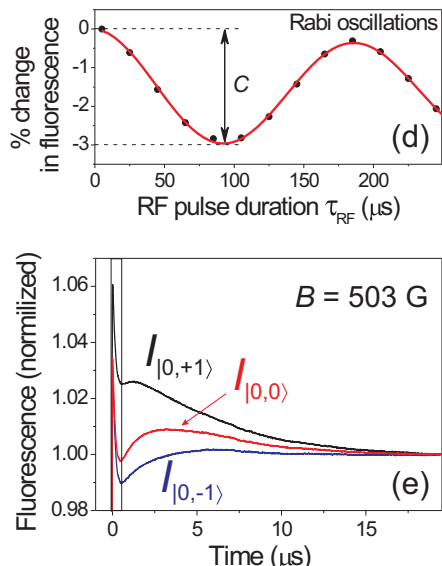

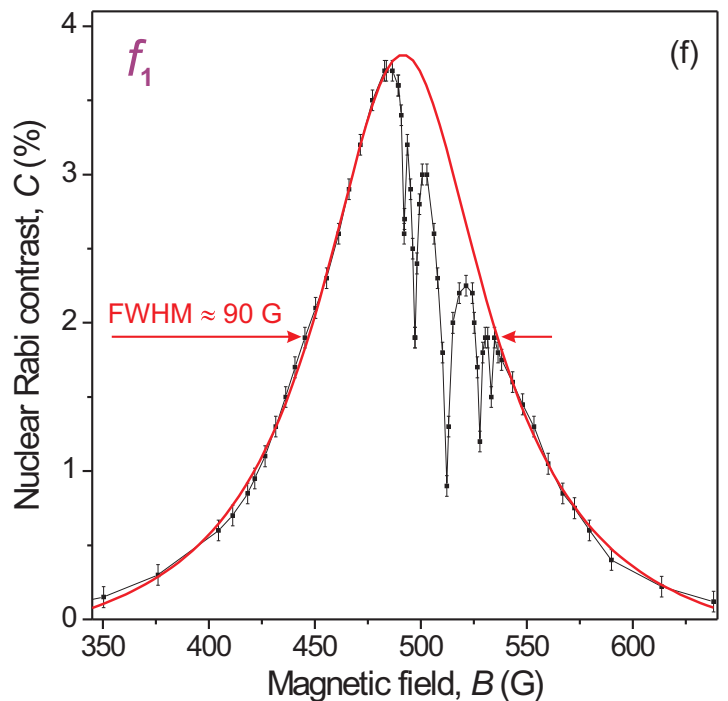

FIG. 2. (a) Experimental pulse sequence used for ODNMR spectroscopy. (b) ODNMR spectrum obtained at a magnetic field $B=503 \mathrm{G}$. The inset illustrates the relative population of nuclear levels at this field. (c) Pulse sequence used to observe nuclear Rabi oscillations. (d) Optically detected nuclear Rabi oscillations for the $f_{1}$ transition. Experimental data (dots) were fitted with an exponentially decaying sine wave (red line) to obtain the maximum oscillation contrast $C$. (e) Fluorescence responses of the $\mathrm{NV}$ centers initialized in $|0,+1\rangle,|0,0\rangle$, and $|0,-1\rangle$ states at $B=503 \mathrm{G}$. The vertical lines at the beginning of the traces show the $0.5 \mu$ s readout time used in the experiments. (f) Contrast of Rabi oscillations between the $|0,+1\rangle$ and $|0,0\rangle$ states as a function of the magnetic field $B$. Experimental data (connected dots with error bars) were fitted with a Lorentzian (red line), whose full width at half maximum (FWHM) was found to be $\sim 90 \mathrm{G}$. Five dips observed in the magnetic field range between 490 and $535 \mathrm{G}$ are due to cross relaxation between NV centers aligned along the field direction and substitutional nitrogen spins in diamond [17].

through a 650-800 nm bandpass filter, and detected with a fiber-coupled $\mathrm{Si}$ avalanche photodiode. Radio-frequency signals were delivered using a $100 \mu \mathrm{m}$ diameter copper wire placed on the diamond surface next to the optical focus. A static magnetic field $B$ was applied along one of the NV axes using a neodymium permanent magnet.

To perform ODNMR spectroscopy, we applied a pulse sequence illustrated in Fig. 2(a). The radio-frequency pulse with a typical duration of $200 \mu$ s was applied between the optical pump and probe pulses, and the fluorescence response of the system was recorded as a function of the radio frequency. Figure 2(b) shows an example of the ${ }^{14} \mathrm{~N}$ ODNMR spectrum recorded at a magnetic field of $B=503 \mathrm{G}$. Two resonances were observed at frequencies $f_{1}$ and $f_{2}$ that correspond to $|0,+1\rangle \rightarrow|0,0\rangle$ and $|0,-1\rangle \rightarrow|0,0\rangle$ transitions, respectively. The amplitudes of the resonances indicate a strong nuclear polarization of the system into the $|0,+1\rangle$ state.

We used a pulse sequence illustrated in Fig. 2(c) to resonantly drive the nuclear spin transition with a radio-frequency pulse of varying duration $\tau_{R F}$. Figure 2(d) shows an example of optically detected nuclear Rabi oscillations between the $|0,+1\rangle$ and $|0,0\rangle$ states, where $C$ is the contrast of Rabi oscillations.

We measured the fluorescence responses of the NV centers selectively initialized in the three nuclear spin states $|0,+1\rangle$, $|0,0\rangle$, and $|0,-1\rangle$ at $503 \mathrm{G}$; see Fig. 2(e). The $|0,+1\rangle$ state was initialized first by optical pumping. The $|0,0\rangle$ state was prepared through transferring the population from the $|0,+1\rangle$ state by applying a radio-frequency $\pi$ pulse resonant with the $f_{1}$ transition. The $|0,-1\rangle$ state was similarly prepared through transferring the population from the $|0,0\rangle$ state by applying a radio-frequency $\pi$ pulse resonant with the $f_{2}$ transition. Nuclear-spin-dependent fluorescence as a function of magnetic field is presented in Appendix B.

Next, we studied the dependence of the resonant nuclear Rabi oscillation contrast $C$ for the $f_{1}$ transition on the applied magnetic field $B$ near the ESLAC; see Fig. 2(f). The contrast of nuclear Rabi oscillations exceeds $2 \%$ from approximately 450 to $550 \mathrm{G}$, reaching its maximum value of $\sim 3.8 \%$ at $\sim 485 \mathrm{G}$. The maximum value of the electron Rabi-oscillation contrast observed for the same sample was $\sim 7 \%$.

\section{MAGNETIC FIELD DEPENDENCE}

Nuclear spin transition frequencies $f_{1}$ and $f_{2}$ were experimentally measured as a function of magnetic field and temperature by implementing a Ramsey interferometry technique. Figures 3(a) and 3(b) depict the pulse timing diagrams for measuring $f_{1}$ and $f_{2}$, respectively. The ${ }^{14} \mathrm{~N} \mathrm{NV}$ nuclear spins are either prepared in the $|0,+1\rangle$ state (to measure $f_{1}$ ) or the $|0,0\rangle$ state (for $f_{2}$ ). Subsequently, a $\pi / 2-\tau-\pi / 2$ pulse sequence is applied. The fluorescence is recorded as a function of $\tau$ and the resulting Ramsey interference fringes, shown in Fig. 3(c), are fit to an exponentially decaying sinusoidal function. From the fit to the Ramsey data, which includes an exponential decay $e^{-\tau / T_{2}^{*}}$, we infer the ${ }^{14} \mathrm{~N}$ nuclear spincoherence time $T_{2}^{*}$. The experimentally measured values of $T_{2}^{*}$ are in the range from 0.5 to $0.8 \mathrm{~ms}$ for all temperature and magnetic field ranges used in this work.

We plot experimental values of $\left(f_{1}+f_{2}\right) / 2$ and $\left(f_{1}-\right.$ $\left.f_{2}\right) / 2 B$ as a function of magnetic field in Figs. 3(d) and 3(e), 

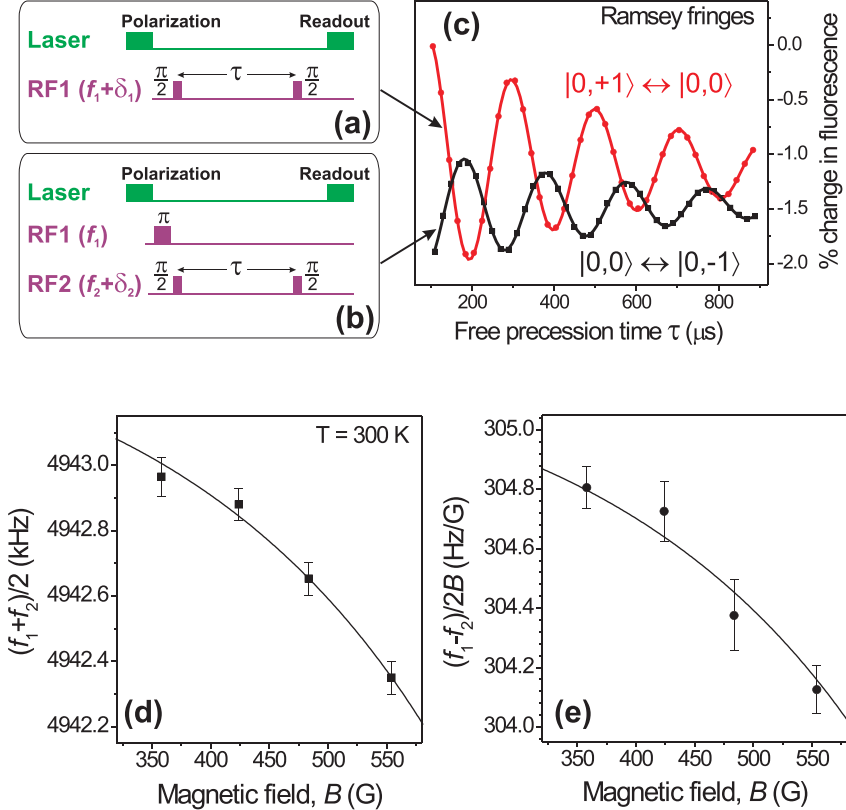

FIG. 3. (a),(b) Experimental pulse sequences used to observe nuclear Ramsey fringes for $f_{1}$ and $f_{2}$ transitions, respectively. The radio-frequency pulse sequence $\pi / 2-\tau-\pi / 2$ with a variable time delay $\tau$ is applied between laser light pulses. $R F 1$ and $R F 2$ are detuned from the resonance frequencies $f_{1}$ and $f_{1}$ by $\delta_{1}$ and $\delta_{2}$, respectively. A $\pi$ pulse resonant with the $f_{1}$ transition is used to prepare nuclear spins in the $|0,0\rangle$ state. (c) Optically detected $\mathrm{NV}{ }^{14} \mathrm{~N}$ nuclear Ramsey fringes for the $f_{1}$ and $f_{2}$ transitions. Symbols represent experimental data, while solid lines are exponentially decaying sine wave fits. The oscillation frequency of the signal corresponds to detuning $\delta$ from the resonance transition $f$, which is $\sim 5 \mathrm{kHz}$ for the presented data. (d) $\left(f_{1}+f_{2}\right) / 2$ and (e) $\left(f_{1}-f_{2}\right) / 2 B$ as a function of $B$. Symbols are values determined from Ramsey spectroscopy, while solid lines are fits to Eqs. (2) and (3), respectively.

respectively. In contrast to those of an ideal nuclear spin, these values are not constant, but decrease with increasing magnetic field strength throughout the studied field range. This is due to mixing of the electron and nuclear spin states via the transverse magnetic hyperfine interaction characterized by the constant $A_{\perp}$. The average value of the nuclear spin transition frequencies, $f_{1}$ and $f_{2}$, is described in (Appendix A)

$$
\frac{f_{1}+f_{2}}{2}=\left|Q+\frac{A_{\perp}^{2} D}{D^{2}-\gamma_{e}^{2} B^{2}}\right| .
$$

The effective nuclear gyromagnetic ratio is determined from

$$
\frac{f_{1}-f_{2}}{2 B}=\gamma_{n}\left(1-\frac{\gamma_{e}}{\gamma_{n}} \frac{A_{\perp}^{2}}{D^{2}-\gamma_{e}^{2} B^{2}}\right),
$$

where $\gamma_{e}$ and $\gamma_{n}$ are the electron and nuclear gyromagnetic ratios, respectively.

We fit the data plotted in Fig. 3(d) to Eq. (2) with the following parameters fixed: $D=2870 \mathrm{MHz}, \gamma_{e}=$ $2.803 \mathrm{MHz} / \mathrm{G}, A_{\perp}=-2.62 \mathrm{MHz}$ [18]. From the fit, we obtained the value of $Q=-4.9457(3) \mathrm{MHz}$ at $T=300 \mathrm{~K}$, which is in agreement with previously reported values $[9,10]$ and represents an order of magnitude improvement in preci-
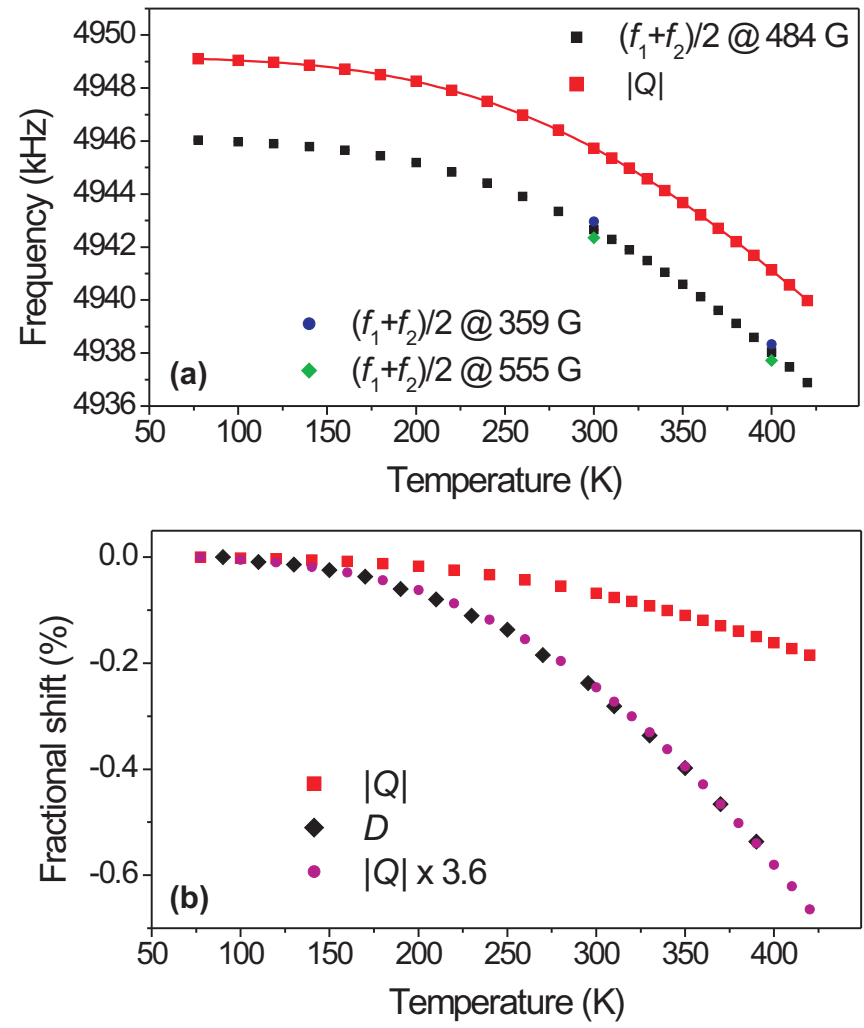

FIG. 4. (a) $\left(f_{1}+f_{2}\right) / 2$ as a function of temperature at $484 \mathrm{G}$ magnetic field applied along the $\mathrm{NV}$ axis. $|Q|$ was determined from the experimental data using Eq. (2). The solid line is a fourth-degree polynomial fit to Eq. (4). (b) Comparison of the fractional shifts of $|Q|$ and $D$ as a function of temperature (Appendix D).

sion. We use Eq. (3) to fit the data in Fig. 3(e) and extract the ${ }^{14} \mathrm{~N}$ gyromagnetic ratio $\gamma_{n}=307.5(3) \mathrm{Hz} / \mathrm{G}$. The obtained value agrees with the literature data [19] and confirms the validity of our theoretical model. Error bars of $Q$ represent the combination of statistical uncertainty and the uncertainty in $A_{\perp}$, while in the case of $\gamma_{n}$, the main uncertainty is associated with that in the magnetic field measurement (Appendix C).

\section{TEMPERATURE DEPENDENCE}

Next, we measure $f_{1}$ and $f_{2}$ as a function of temperature and use Eq. (2) to determine $Q(T)$. The term $A_{\perp}^{2} D /\left(D^{2}-\right.$ $\gamma_{e}^{2} B^{2}$ ) in Eq. (2) is $\sim 3 \mathrm{kHz}$ at $480 \mathrm{G}$, which is $\sim 0.06 \%$ of $Q$. Therefore, the temperature dependence of $D$ has a negligible effect on $Q(T)$. Nevertheless, we used experimental values of $D(T)$ (Appendix D) in order to obtain more accurate values for $Q(T)$. The temperature dependence of $A_{\perp}$ is not known, but if we assume that its fractional temperature dependence is similar to that of $D$, then $A_{\perp}(T)$ also has a negligible effect on $Q(T)$. We use a constant value of $A_{\perp}=-2.62 \mathrm{MHz}$ throughout the entire temperature range. Figure 4(a) shows the experimentally measured values of $\left(f_{1}+f_{2}\right) / 2$ and the inferred value of $|Q|$ from Eq. (2) as a function of temperature. $|Q|$ is shifted from $\left(f_{1}+f_{2}\right) / 2$ by $\sim 3 \mathrm{kHz}$ and smoothly decreases by $\sim 10 \mathrm{kHz}$ with temperature increasing from 77.5 to $420 \mathrm{~K}$. 
We fit the experimentally determined $|Q|$ data to the fourthorder polynomial function,

$$
|Q(T)|=\sum_{n=0}^{4} a_{n} T^{n} .
$$

The fit values of the coefficients are $a_{0}=4949.473 \mathrm{kHz}, a_{1}=$ $-9.32 \times 10^{-3} \mathrm{kHz} / \mathrm{K}, \quad a_{2}=9.2597 \times 10^{-5} \mathrm{kHz} / \mathrm{K}^{2}, a_{3}=$ $-4.6294 \times 10^{-7} \mathrm{kHz} / \mathrm{K}^{3}$, and $a_{4}=3.983 \times 10^{-10} \mathrm{kHz} / \mathrm{K}^{4}$. The temperature slope of the nuclear quadrupole coupling constant $Q$ at $297 \mathrm{~K}$ is $d|Q| / d T=-35.0(2) \mathrm{Hz} / \mathrm{K}$, which is $\sim 2000$ times smaller than the temperature dependence of the zero-field splitting parameter $D$ of electron spin transitions [12], which is $d D / d T=-74.2(7) \mathrm{kHz} / \mathrm{K}$. A recent paper [20] used a different technique to infer a value of $d|Q| / d T=$ $-24(4) \mathrm{Hz} / \mathrm{K}$ that is lower than our result, but with an order of magnitude larger uncertainty.

We find that the fractional changes of $Q$ and $D$ as a function of temperature match almost perfectly up to a constant factor of 3.6 [see Fig. 4(b)]. This may suggest that the temperature dependence of $Q$ and $D$ arises from a common mechanism. Such a mechanism that would cause similar changes in $Q$ and $D$ is not obvious because $Q$ and $D$ arise from different interactions that depend on different aspects of the NV center's electron orbitals. $D$ arises from the magnetic dipolar interactions between the NV center's two unpaired electrons, while $Q$ arises from the interaction between the ${ }^{14} \mathrm{~N}$ electric quadrupole moment and the electric-field gradient at the nucleus.

The equivalent temperature variations of $D$ and $Q$ present an interesting theoretical problem that will potentially reveal new microscopic understanding of the NV center. Accordingly, it should be pursued in the future with ab initio calculations. The discussions presented in Appendix E may serve as an intuition to guide those calculations.

\section{CONCLUSION AND OUTLOOK}

In this work, motivated by the development of diamondbased rotation sensors, we investigated the nonlinear temperature and magnetic field dependence of the ${ }^{14} \mathrm{~N}$ hyperfine spin transitions in an ensemble of diamond NV centers. These measurements were enabled by a direct optical readout technique (without the use of microwave transitions) optimized in this work. The fluorescence contrast of nuclear Rabi oscillations depends on magnetic field and reaches its maximum value of $\sim 3.8 \%$ at around $485 \mathrm{G}$. Such a high contrast is comparable to that of the electron spin transitions.

From the magnetic field dependence of the frequencies of the nuclear spin transition, we determine the values of the nuclear quadruple coupling constant $Q$, and gyromagnetic ratio $\gamma_{n}$ for $\mathrm{NV}{ }^{14} \mathrm{~N}$, which are in agreement with the published values.

While the measured temperature dependence of the nuclear spin transition is smaller than the corresponding dependence of the electron spin transitions in both absolute (by a factor of $\sim 2000$ ) and relative (by a factor of $\sim 3.6$ ) measure, this temperature dependence can still prove problematic for precision sensors. This dependence can be further reduced by reconfiguring the measurement to sense the interval between the
$m_{I}= \pm 1$ levels directly, in analogy with how this is done for electronic states [21]. Another approach is to use spin- $1 / 2{ }^{15} \mathrm{~N}$ nuclei instead of ${ }^{14} \mathrm{~N}$ that do not have a quadrupole moment and therefore there is no quadrupole splitting. The relative advantages and disadvantages of using ${ }^{15} \mathrm{~N}$ vs ${ }^{14} \mathrm{~N}$ centers for gyroscopic applications require separate consideration.

\section{ACKNOWLEDGMENTS}

The authors are grateful to Chih-Wei Lai, Pauli Kehayias, and Sean Lourette for useful discussions. A.J. acknowledges support from the U.S. Army Research Laboratory under Cooperative Agreement No. W911NF-16-2-0008. I.F. and V.M.A. were supported by a Cottrell Scholars Award. This work was supported in part by EU FET-OPEN Flagship Project ASTERIQS (Action No. 820394) and the German Federal Ministry of Education and Research (BMBF) within the Quantumtechnologien program (Grant No. FKZ 13N14439). M.W.D. acknowledges support from the Australian Research Council (Grant No. DE170100169).

\section{APPENDIX A: NV GROUND-STATE TRANSITIONS}

The relevant spin Hamiltonian of the NV ground state in the presence of an axial magnetic field $B_{z}$ can be written as

$$
\begin{aligned}
H= & D\left(S_{z}^{2}-\frac{1}{3} \mathbf{S}^{2}\right)+\gamma_{e} S_{z} B_{z}+Q\left(I_{z}^{2}-\frac{1}{3} \mathbf{I}^{2}\right) \\
& -\gamma_{n} I_{z} B_{z}+A_{\|} S_{z} I_{z}+\frac{A_{\perp}}{2}\left(S_{+} I_{-}+S_{-} I_{+}\right),
\end{aligned}
$$

where $\mathbf{S}$ and $\mathbf{I}$ are the dimensionless electron and nuclear spin operators, respectively, $D$ is the zero-field electron spin-spin interaction, $\gamma_{e}$ and $\gamma_{n}$ are the electron and nuclear gyromagnetic ratios, respectively, $Q$ is the nuclear quadrupole coupling constant, and $A_{\|}$and $A_{\perp}$ are the axial and transverse magnetic hyperfine constants. Treating the transverse magnetic hyperfine interaction as a perturbation, the nuclear spin Hamiltonian of the $m_{S}=0$ manifold is, to second order,

$$
\begin{aligned}
H_{0}= & \left(Q+\frac{A_{\perp}^{2} D}{D^{2}-\gamma_{e}^{2} B_{z}^{2}}\right)\left(I_{z}^{2}-\frac{2}{3}\right) \\
& -\gamma_{n}\left(1-\frac{\gamma_{e}}{\gamma_{n}} \frac{A_{\perp}^{2}}{D^{2}-\gamma_{e}^{2} B_{z}^{2}}\right) I_{z} B_{z},
\end{aligned}
$$

from which we can determine the average value of the nuclear spin transitions $f_{1}$ and $f_{2}$,

$$
\frac{f_{1}+f_{2}}{2}=\left|Q+\frac{A_{\perp}^{2} D}{D^{2}-\gamma_{e}^{2} B_{z}^{2}}\right|,
$$

and the effective nuclear gyromagnetic ratio $\gamma_{n}^{\text {eff }}$,

$$
\gamma_{n}^{\text {eff }}=\frac{f_{1}-f_{2}}{2 B_{z}}=\gamma_{n}\left(1-\frac{\gamma_{e}}{\gamma_{n}} \frac{A_{\perp}^{2}}{D^{2}-\gamma_{e}^{2} B_{z}^{2}}\right) .
$$

These expressions are valid sufficiently far from the ground-state level anticrossing (GSLAC), where the term with the resonant denominator is a small correction. We also note in passing that in the vicinity of the GSLAC, there is an interesting regime to study the temperature and magnetic field dependence of $A_{\perp}$, which will be a subject of future work. 

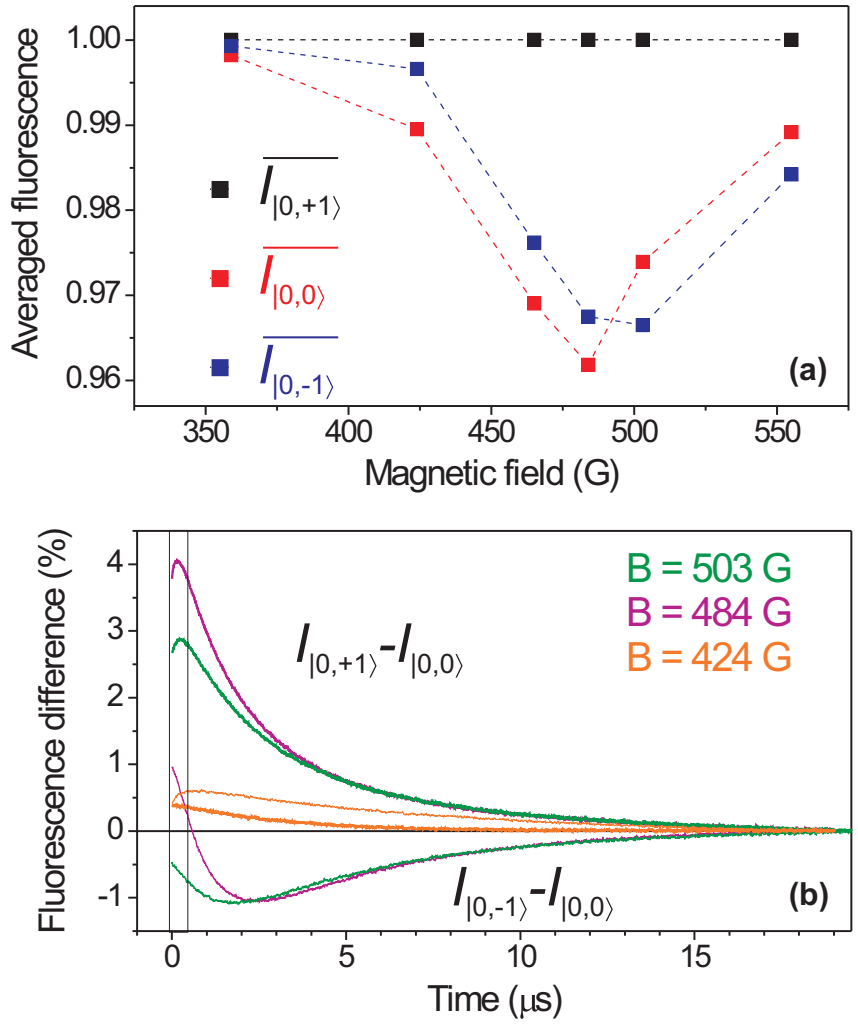

FIG. 5. (a) Normalized averaged nuclear spin states' fluorescence responses $(0.5 \mu$ s readout time $)$ as a function of magnetic field. (b) Nuclear spin states' fluorescence response differences at the selected magnetic field strengths (thicker lines: $I_{|0,+1\rangle}-I_{|0,0\rangle}$; thinner lines: $\left.I_{|0,-1\rangle}-I_{|0,0\rangle}\right)$. The vertical lines at the beginning of the traces show the $0.5 \mu$ s readout time used in the experiments presented in the main text.

\section{APPENDIX B: NUCLEAR-SPIN-DEPENDENT FLUORESCENCE}

Figure 5(a) shows averaged and normalized fluorescence responses $(0.5 \mu$ s readout time) of the $\mathrm{NV}$ centers selectively initialized in the three nuclear spin states of the $m_{S}=0$ manifold $(|0,+1\rangle,|0,0\rangle$, and $|0,-1\rangle)$ as a function of magnetic field. The fluorescence rate for the $|0,+1\rangle$ state is the highest for the studied magnetic field range, while the relative fluorescence rate for the $|0,0\rangle$ and $|0,-1\rangle$ states depends on the magnetic field strength. The fluorescence rate for the $|0,-1\rangle$ state is higher than that for the $|0,0\rangle$ state at the magnetic field strength below $\approx 495 \mathrm{G}$ and it is lower at magnetic field strength above $\approx 495 \mathrm{G}$. This relative fluorescence-response behavior reflects the degree of mixing in the excited state depending on the magnetic field. Figure 5(b) demonstrates differences in the fluorescence responses for the selectively initialized nuclear spin states $|0,+1\rangle,|0,0\rangle$, and $|0,-1\rangle$ at 503, 484, and $424 \mathrm{G}$.

\section{APPENDIX C: MAGNETIC FIELD ALIGNMENT AND CALIBRATION}

A static magnetic field $\mathbf{B}$ was applied along the NV axis using a neodymium permanent magnet mounted on a three-
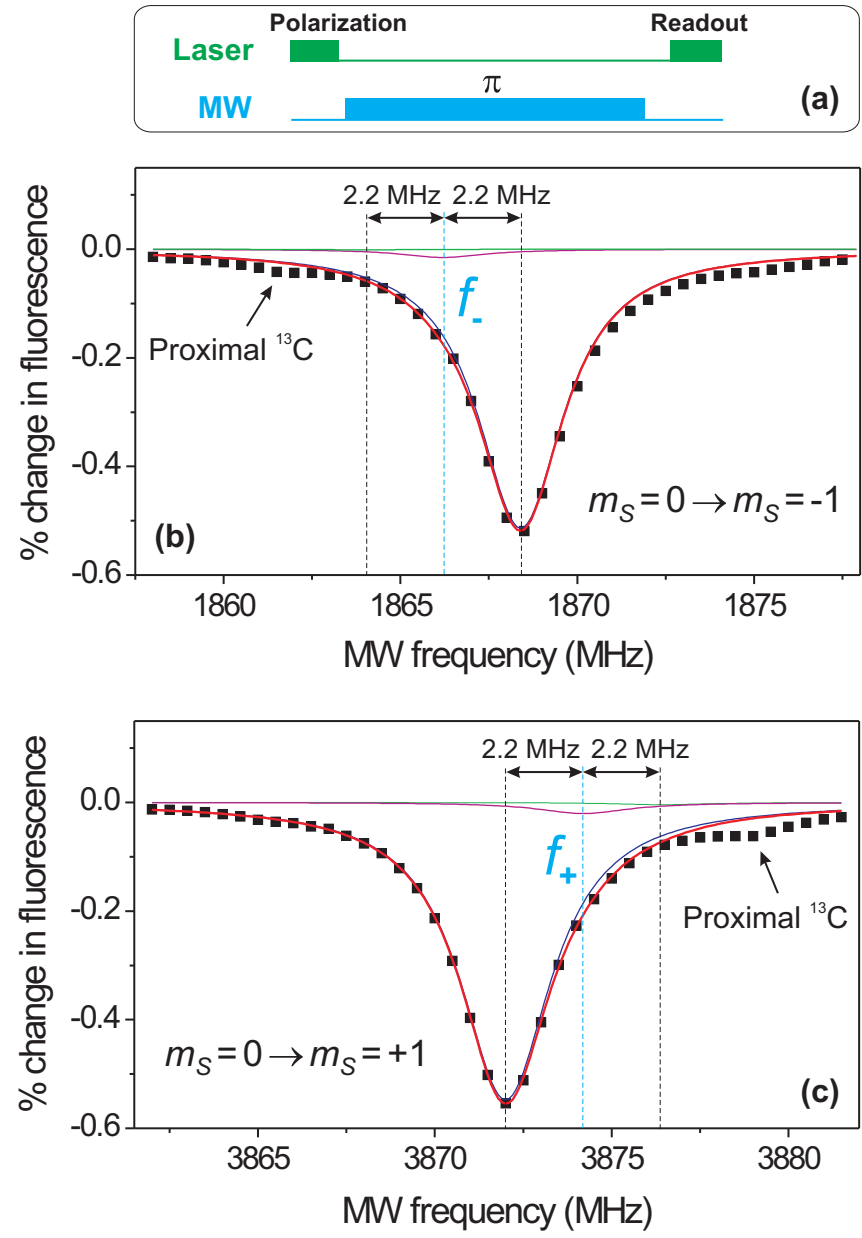

FIG. 6. (a) Experimental pulse sequence used to obtain ODMR signals. (b),(c) Pulsed ODMR signals for the NV subensembles aligned along the magnetic field $\mathbf{B}$ for $m_{S}=0 \rightarrow m_{S}=-1$ and $m_{S}=0 \rightarrow m_{S}=+1$ transitions, respectively.

axis translation stage. The alignment of the applied magnetic field was done by overlapping three NV electron spin resonances corresponding to the three NV subensembles, which are not aligned with the field. The alignment of the $\mathbf{B}$ along the [111] axis is estimated to be better than 0.2 degree. The electron spin resonances associated with the NV subensemble aligned with $\mathbf{B}$ were used to determine the strength of the applied field. Pulsed optically detected magnetic resonance (ODMR) signals were recorded for both $m_{S}=0 \rightarrow m_{S}=-1$ and $m_{S}=0 \rightarrow m_{S}=+1$ transitions in Fig. 6. The duration of the microwave $\pi$ pulse in the pulsed ODMR measurement was of the order of $2 \mu \mathrm{s}$. Each ODMR signal was fitted with the three Lorentzians separated by $2.2 \mathrm{MHz}$. The relative amplitudes of the Lorentzians' fit indicates the high degree of nuclear spin polarization, $>95 \%$, even at $\approx 150 \mathrm{G}$ away from the ESLAC. We used the fit values of the central Lorentzian frequency for both transitions $f_{-}$and $f_{+}$to determine the strength of the magnetic field,

$$
B=\frac{f_{+}-f_{-}}{2 \gamma_{e}},
$$

where $\gamma_{e}=2.803 \mathrm{MHz} / \mathrm{G}$. We estimate the statistical uncertainty of the magnetic field measurement to be better than 


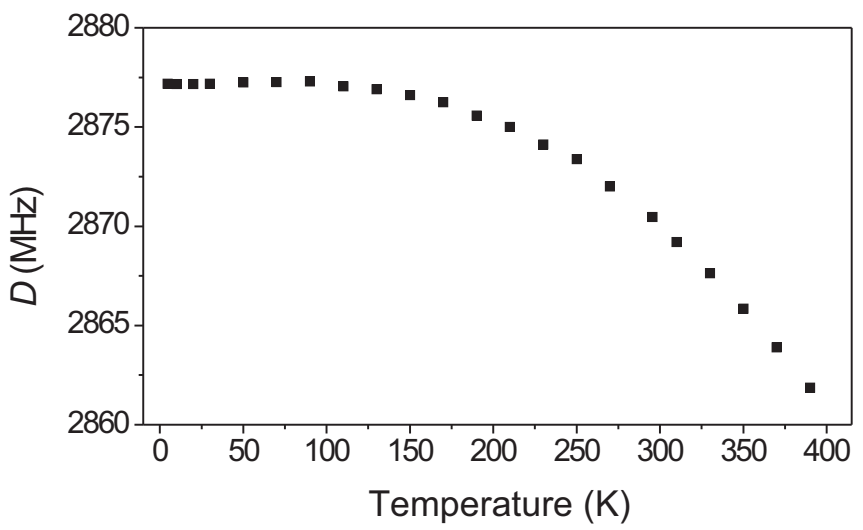

FIG. 7. Experimental values of zero-field splitting $D$ as a function of temperature.

$0.1 \mathrm{G}$. Nevertheless, the drift of the magnetic field during the nuclear Ramsey measurements can introduce a systematic uncertainty in $\mathbf{B}$. This magnetic field drift associated with the permanent magnet's sensitivity to the ambient temperature fluctuations represents the main source of uncertainty in the measurement of the nuclear gyromagnetic ratio $\gamma_{n}$. We estimate the maximum systematic error introduced by the magnetic field drift to be less than $0.3 \mathrm{G}$. In the ODMR signal, we also observe polarization of the ${ }^{13} \mathrm{C}$ nuclear spins proximal to the $\mathrm{NV}$ centers with hyperfine interaction strength of 13-14 MHz [16].

\section{APPENDIX D: TEMPERATURE DEPENDENCE OF $D$}

Figure 7 shows the experimentally measured values of $D$ as a function of temperature for a diamond sample similar to that used in the present work $([\mathrm{N}] \approx 50 \mathrm{ppm},[\mathrm{NV}] \approx 16 \mathrm{ppm})$ in the range from 5 to $390 \mathrm{~K}$. These results were obtained employing a standard ODMR technique. The data for the temperature range from 5 to $300 \mathrm{~K}$ were published in [22], while the rest of the data remain unpublished. We use this data in Eq. (2) to extract $Q$ as a function of temperature from experimentally measured values $f_{1}$ and $f_{2}$, as well as to plot the fractional shift of $D$ in Fig. 4(b) of the main text in the range from 90 to $390 \mathrm{~K}$. Other studies of $D(T)$ in the temperature range of 5.6-295 K was reported in Ref. [23] and in the temperature range of 300-700 K in Ref. [24].

\section{APPENDIX E: $D(T)$ VS $Q(T)$}

To find some link between $D$ and $Q$, we need to also consider how $D$ depends on individual atomic orbitals. As shown in Ref. [25], $D$ is approximately determined by the separation of the mean positions of electrons in the dangling $s p^{3}$ carbon orbitals around the vacancy. These mean positions depend on the carbon lattice positions and the $s p$ hybridization of their dangling orbitals. The lattice positions and hybridization are correlated because if the carbons move relative to their nearest neighbors, then their atomic orbitals must rehybridize to maintain bonds with those neighbors (this is often called bond bending). The same is true of the hybridization of the nitrogen atom's orbitals and so the occupation of the nitrogen's $p_{z}$ orbital is correlated to the nitrogen's lattice position. Thus, $D$ and $Q$ are linked if there is a common displacement (up to a proportionality factor) of the carbon and nitrogen atoms around the vacancy.

As shown in Ref. [22], the temperature dependence of $D$ has two contributions: thermal expansion and quadratic electron-phonon interactions. These contributions are generalizable across the different types of resonances of the NV center (i.e., visible and infrared) [22] and so are also expected to govern the temperature dependence of $Q$ (although in that case it will be nuclear-phonon interactions). Both of these contributions represent lattice displacements: either static (thermal expansion) or dynamic (interactions with phonons). Hence, if there are common displacements of the nitrogen and carbon atoms, then this may result in the qualitatively identical temperature variations of $D$ and $Q$.
[1] M. W. Doherty, N. B. Manson, P. Delaney, F. Jelezko, J. Wrachtrup, and L. C. L. Hollenberg, The nitrogen-vacancy color center in diamond, Phys. Rep. 528, 1 (2013).

[2] L. Rondin, J.-P. Tetienne, T. Hingant, J.-F. Roch, P. Maletinsky, and V. Jacques, Magnetometry with nitrogenvacancy defects in diamond, Rep. Prog. Phys. 77, 056503 (2014).

[3] C. L. Degen, F. Reinhard, and P. Cappellaro, Quantum sensing, Rev. Mod. Phys. 89, 035002 (2017).

[4] J. F. Barry, J. M. Schloss, E. Bauch, M. J. Turner, C. A. Hart, L. M. Pham, and R. L. Walsworth, Sensitivity optimization for NV-diamond magnetometry, Rev. Mod. Phys. 92, 015004 (2020).

[5] M. P. Ledbetter, K. Jensen, R. Fischer, A. Jarmola, and D. Budker, Gyroscopes based on nitrogen-vacancy centers in diamond, Phys. Rev. A 86, 052116 (2012).

[6] A. Ajoy and P. Cappellaro, Stable three-axis nuclear-spin gyroscope in diamond, Phys. Rev. A 86, 062104 (2012).
[7] D. Maclaurin, M. W. Doherty, L. C. L. Hollenberg, and A. M. Martin, Measurable Quantum Geometric Phase from a Rotating Single Spin, Phys. Rev. Lett. 108, 240403 (2012).

[8] J.-C. Jaskula, K. Saha, A. Ajoy, D. J. Twitchen, M. Markham, and P. Cappellaro, Cross-Sensor Feedback Stabilization of an Emulated Quantum Spin Gyroscope, Phys. Rev. Appl. 11, 054010 (2019).

[9] B. Smeltzer, J. McIntyre, and L. Childress, Robust control of individual nuclear spins in diamond, Phys. Rev. A 80, 050302(R) (2009).

[10] M. Steiner, P. Neumann, J. Beck, F. Jelezko, and J. Wrachtrup, Universal enhancement of the optical readout fidelity of single electron spins at nitrogen-vacancy centers in diamond, Phys. Rev. B 81, 035205 (2010).

[11] P. Neumann, J. Beck, M. Steiner, F. Rempp, H. Fedder, P. R. Hemmer, J. Wrachtrup, and F. Jelezko, Single-shot readout of a single nuclear spin, Science 329, 542 (2010). 
[12] V. M. Acosta, E. Bauch, M. P. Ledbetter, A. Waxman, L.-S. Bouchard, and D. Budker, Temperature Dependence of The Nitrogen-Vacancy Magnetic Resonance in Diamond, Phys. Rev. Lett. 104, 070801 (2010).

[13] J. S. Hodges, N. Y. Yao, D. Maclaurin, C. Rastogi, M. D. Lukin, and D. Englund, Timekeeping with electron spin states in diamond, Phys. Rev. A 87, 032118 (2013).

[14] N. B. Manson, J. P. Harrison, and M. J. Sellars, Nitrogenvacancy center in diamond: Model of the electronic structure and associated dynamics, Phys. Rev. B 74, 104303 (2006).

[15] V. Jacques, P. Neumann, J. Beck, M. Markham, D. Twitchen, J. Meijer, F. Kaiser, G. Balasubramanian, F. Jelezko, and J. Wrachtrup, Dynamic Polarization of Single Nuclear Spins by Optical Pumping of Nitrogen-Vacancy Color Centers in Diamond at Room Temperature, Phys. Rev. Lett. 102, 057403 (2009).

[16] R. Fischer, A. Jarmola, P. Kehayias, and D. Budker, Optical polarization of nuclear ensembles in diamond, Phys. Rev. B 87, 125207 (2013).

[17] L. T. Hall, P. Kehayias, D. A. Simpson, A. Jarmola, A. Stacey, D. Budker, and L. C. L. Hollenberg, Detection of nanoscale electron spin resonance spectra demonstrated using nitrogenvacancy center probes in diamond, Nat. Commun. 7, 10211 (2016).

[18] Q. Chen, I. Schwarz, F. Jelezko, A. Retzker, and M. B. Plenio, Optical hyperpolarization of ${ }^{13} \mathrm{C}$ nuclear spins in nanodiamond ensembles, Phys. Rev. B 92, 184420 (2015).

[19] R. K. Harris, E. D. Becker, S. M. C. de Menezes, R. Goodfellow, and P. Granger, NMR nomenclature: Nuclear spin properties and conventions for chemical shifts: Iupac recommendations 2001, Solid State Nucl. Magn. Reson. 22, 458 (2002).

[20] V. V. Soshenko, V. V. Vorobyov, O. Rubinas, B. Kudlatsky, A. I. Zeleneev, S. V. Bolshedvorskii, V. N. Sorokin, A. N. Smolyaninov, and A. V. Akimov, Temperature drift rate for nuclear terms of $\mathrm{NV}$ center ground state Hamiltonian, arXiv:1807.08100.

[21] K. Fang, V. M. Acosta, C. Santori, Z. Huang, K. M. Itoh, H. Watanabe, S. Shikata, and R. G. Beausoleil, High-Sensitivity Magnetometry Based on Quantum Beats in Diamond NitrogenVacancy Centers, Phys. Rev. Lett. 110, 130802 (2013).

[22] M. W. Doherty, V. M. Acosta, A. Jarmola, M. S. J. Barson, N. B. Manson, D. Budker, and L. C. L. Hollenberg, Temperature shifts of the resonances of the $\mathrm{NV}^{-}$center in diamond, Phys. Rev. B 90, 041201(R) (2014).

[23] X.-D. Chen, C.-H. Dong, F.-W. Sun, C.-L. Zou, J.-M. Cui, Z.-F. Han, and G.-C. Guo, Temperature dependent energy level shifts of nitrogen-vacancy centers in diamond, Appl. Phys. Lett. 99, 161903 (2011).

[24] D. M. Toyli, D. J. Christle, A. Alkauskas, B. B. Buckley, C. G. Van de Walle, and D. D. Awschalom, Measurement and Control of Single Nitrogen-Vacancy Center Spins Above 600 K, Phys. Rev. X 2, 031001 (2012).

[25] M. W. Doherty, V. V. Struzhkin, D. A. Simpson, L. P. McGuinness, Y. Meng, A. Stacey, T. J. Karle, R. J. Hemley, N. B. Manson, L. C. L. Hollenberg, and S. Prawer, Electronic Properties and Metrology Applications of the Diamond $\mathrm{NV}^{-}$ Center Under Pressure, Phys. Rev. Lett. 112, 047601 (2014). 